\title{
Uso de hormigones con fibras de ultra-alta resistencia para el desarrollo de conexiones entre elementos prefabricados
}

\author{
Ultra High Performance Fibre Reinforced Concrete \\ (UHPFRC) for connections between precast elements
}

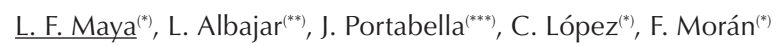

RESUMEN

El adecuado comportamiento de estructuras prefabricadas de hormigón se encuentra condicionado por el diseño y ejecución de las conexiones entre sus elementos. El uso de conexiones rígidas basadas en la adherencia mediante el solape de armaduras se ve en muchos casos limitado, a pesar de su simplicidad, por problemas derivados de las considerables longitudes de solape requeridas y la congestión en el refuerzo. En el desarrollo de este estudio, enmarcado dentro del trabajo de investigación para la puesta a punto de una conexión para pórticos prefabricados con el uso de hormigones de altas especificaciones reforzados con fibras (Ultra High Performance Fibre Reinforced Concrete-[UHPFRC]), se evaluó experimentalmente el comportamiento de solapes de armaduras embebidos en UHPFRC en conexiones simples de vigas de hormigón armado. Elementos viga biapoyados construidos a partir de dos medias vigas prefabricadas unidos mediante el solape de armaduras en una zona central hormigonada con UHPFRC y sin el uso de cercos cerrados o tirantes verticales de refuerzo trasversal fueron ensayados a flexión. Los resultados experimentales para solapes con longitudes entre $10 \mathrm{db}$ y $20 \mathrm{db}$ indican un comportamiento satisfactorio para las conexiones sometidas a ciclos de carga y descarga, condición desfavorable para las condiciones de adherencia, y con recubrimientos y separación trasversal entre solapes escasa, inferior a 0,7 db. Se evidencia la influencia de la mayor resistencia a tracción y ductilidad de los UHPFRC en el confinamiento, las condiciones de adherencia, la transferencia de tensiones y su efecto rigidizador.

Palabras clave: prefabricados, conexión, adherencia, solape, splitting, UHPFRC.
SUMMARY

The performance suitability of precast concrete structures is determined by the design and construction of connections between elements. In spite of its simplicity, the use of bond connections through bar reinforcement splices is limited by issues related to long splice lengths required and reinforcement congestion. Reinforcement splices embedded in Ultra High Performance Fibre Reinforced Concrete (UHPFRC) were tested in beam specimens, as part of an on-going investigation to develop a novel typology of beam-column connection between precast elements using UHPFRC. Elements made up of two precast elements joined through reinforcement splices in a central region poured with UHPFRC, and without using closed stirrups or vertical ties were tested in beam tests. The experimental results for splice lengths between $10 \mathrm{db}$ and $20 \mathrm{db}$ exhibited an adequate performance to connections undergoing load and unload cycles, being this an unfavourable condition for bond capacity, and using short covers and transversal spacing between splices, under $0.7 \mathrm{db}$. It is therefore clear that the higher tensile capacity and ductility of UHPFRC have a positive effect on confinement, bond conditions and force transference as well as a stiffness effect.

Keywords: precast, connection, bond, splice, splitting, UHPFRC.
Informes de la Construcción

Vol. 62, 520, 27-41, octubre-diciembre 2010 ISSN: 0020-0883

elSSN: 1988-3234

doi: 10.3989/ic.09.062

\footnotetext{
(*Instituto de Ciencias de la Construcción Eduardo Torroja, IETcC-CSIC, Madrid (España)

${ }^{(* *}$ E.T.S.I.C.C.P., Universidad Politécnica de Madrid, Madrid (España)

${ }^{(* *)}$ PRAINSA

Persona de contacto/Corresponding author: femayadu@gmail.com (L. F. Maya)
} 


\section{INTRODUCCIÓN}

El comportamiento del hormigón armado obedece a la efectividad de los mecanismos de transferencia de carga entre los materiales que lo constituyen, hormigón y acero. Es el mecanismo de adherencia el que permite, en gran medida, la transferencia de cargas entre el acero de refuerzo y la masa de hormigón que le rodea. Las flechas, anchos de fisuras, resistencia y ductilidad estructural de elementos bajo carga monótona, o la capacidad de absorción y disipación de energía bajo regímenes de carga no estáticos están directa o indirectamente relacionados con la adherencia en los estados límites de servicio y agotamiento. Igualmente es éste el mecanismo responsable de activar la colaboración del hormigón en tracción en elementos de hormigón reforzados, efecto denominado tension-stiffening.

Dada la importancia de la adherencia, la mayoría de los códigos de diseño procuran prevenir la ocurrencia de fallos de adherencia que comprometan el comportamiento estructural mediante el establecimiento de recomendaciones para las longitudes de anclaje y solape de barras embebidas en hormigón. Los principales parámetros de diseño en este sentido están relacionados con el recubrimiento de hormigón, el diámetro de la barra, la longitud del anclaje o del solape, la separación trasversal y longitudinal de los solapes, la resistencia del hormigón y el confinamiento.

En el desarrollo de estructuras prefabricadas, donde la fabricación en planta permite un mayor control de la calidad de los elementos, son las conexiones las que constituyen el factor principal para garantizar un adecuado comportamiento de las estructura, siendo ellas además la parte del proceso constructivo que se realiza in situ durante el cual se requiere dar solución a los inconvenientes usualmente presentes en el montaje de la estructura.

El uso de conexiones por adherencia mediante el solape de armaduras, a pesar de su simplicidad, se ve, en muchos casos, limitado por problemas en el diseño de detalle de los elementos derivados de las considerables longitudes de solape requeridas.

El desarrollo de este estudio, correspondiente a la primera fase de un trabajo de investigación para la puesta a punto de conexiones en pórticos prefabricados, se concentra en la evaluación del uso de UHPFRC por sus siglas en ingles, para el solape de armaduras en conexiones simples de hormigón armado.

\section{ANTECEDENTES}

Se diferencian dos tipos de fallo de adherencia entre las barras de acero y el hormigón; el fallo por arrancamiento o pullout y el fallo por propagación de fisuras longitudinales o splitting, siendo este término equivalente al término desgarramiento o hendimiento utilizado en castellano en el Código Modelo CEB-FIP 1990 (1). Se utilizarán sin embargo los términos en inglés debido a su comprensión sin equívocos y aceptación general por parte de los investigadores.

El fallo por pullout es debido principalmente a la formación de una superficie de corte en el hormigón entre dos corrugas, se relaciona con un mecanismo local de fallo por colapso de la interfase. El fallo por splitting se debe principalmente a la fisuración longitudinal del hormigón que rodea el refuerzo y la pérdida de la adherencia una vez que las fisuras alcanzan la superficie del elemento, se relaciona el fallo con un colapso estructural ya que están involucrados otros parámetros estructurales diferentes a los conectados con la adherencia (2). El control del fallo por splitting se aborda en normas y recomendaciones de diseños mediante prescripciones para el recubrimiento y la separación longitudinal y transversal de los solapes.

Uno de los medios más funcionales para mejorar la capacidad adherente de las barras de acero de refuerzo embebidas en hormigón es el confinamiento. En la práctica, el confinamiento se obtiene básicamente mediante la disposición de refuerzo trasversal que, sin embargo, debido a los ajustados espaciamientos, origina, en muchas ocasiones, congestión y dificultades en el hormigonado de las secciones. El uso de hormigones reforzados con fibras, FRC, por sus siglas en inglés, ha ganado en los últimos años un considerable interés en la sustitución parcial del refuerzo trasversal, aportando el confinamiento requerido y facilitando las labores constructivas. Se han incorporado una amplia variedad de fibras con distintas propiedades físicas y mecánicas de materiales como acero, vidrio, carbono, polipropileno, aramida y con formas y geometrías lisas, corrugadas, rectas, deformadas, planas, abultadas, entre otras muchas.

Los porcentajes de fibras usualmente incorporados, inferiores al 2,0\%, si bien no aportan incrementos demasiados significativos en la capacidad resistente de elementos a tracción o flexión, sí presentan, debido a la deformación de rotura de las fibras superior en dos o tres órdenes de magnitud a la de la matriz cementante, un mejor comportamiento post-fisuración con incrementos en la deformación de rotura y la ductilidad. Su 
efecto en las características de adherencia del material y la resistencia de anclajes y solapes de armadura parece ser subestimado con la realización de ensayos de pullout (3), lográndose ligeros o insignificantes aumentos en las tensiones pico de adherencia pero evidenciando el efecto de la mayor ductilidad en el momento de fallo. La realización de ensayos de solapes de armaduras en vigas ha permitido observar aumentos significativos en las tensiones de adherencia y la resistencia al splitting (4), representando las condiciones de adherencia esperadas en los elementos en la práctica de mejor forma.

Más recientemente, el uso de UHPFRC ha resultado efectivo en el aumento de la capacidad de deformación y la reducción de daño en cierto tipo de estructuras (5). En general, los UHPFRC son materiales con gran compacidad de la matriz y altos contenidos de fibras que se caracterizan por tener un comportamiento a tracción dúctil con strain-hardening y una fisuración múltiple no localizada, Figura 1. El uso de estos materiales favorece igualmente la durabilidad de las estructuras debido al menor ancho de las fisuras y la compacidad de la matriz que limitan la entrada y circulación de agentes agresivos.

La información disponible sobre el anclaje y solape de barras de refuerzo en UHPFRC es todavía limitada. Sin embargo, dado el comportamiento referido para estos materiales, una mayor compatibilidad de deformaciones entre la matriz y el refuerzo puede manifestarse incluso hasta alcanzar la plastificación del acero (6). La consideración de deslizamientos relativos entre el acero y el HPFRCC, para niveles de solicitación generalmente superiores a las solicitaciones de servicio, llevan a considerar un comportamiento adherente inicialmente más rígido, con altas tensiones de adherencia para pequeños deslizamientos, consecuente con la mayor resistencia a tracción del material, y una mayor ductilidad derivada del incremento del confinamiento por la acción de las fibras. Pero no es sólo la adición de grandes cantidades de fibras sino también la compacidad de la matriz cementante la responsable de la mejora de las condiciones de adherencia del refuerzo, como se desprende de los resultados obtenidos por Klotz et al. (7) para hormigones de alta resistencia, UHPC, fabricados mediante el concepto de polvos reactivos utilizado en la mayoría de los UHPFRC

Las limitaciones impuestas en la concepción de estructuras prefabricadas por las estrictas longitudes de solape requeridas con hormigones y morteros estándar, han estimulado la evaluación de soluciones alternativas

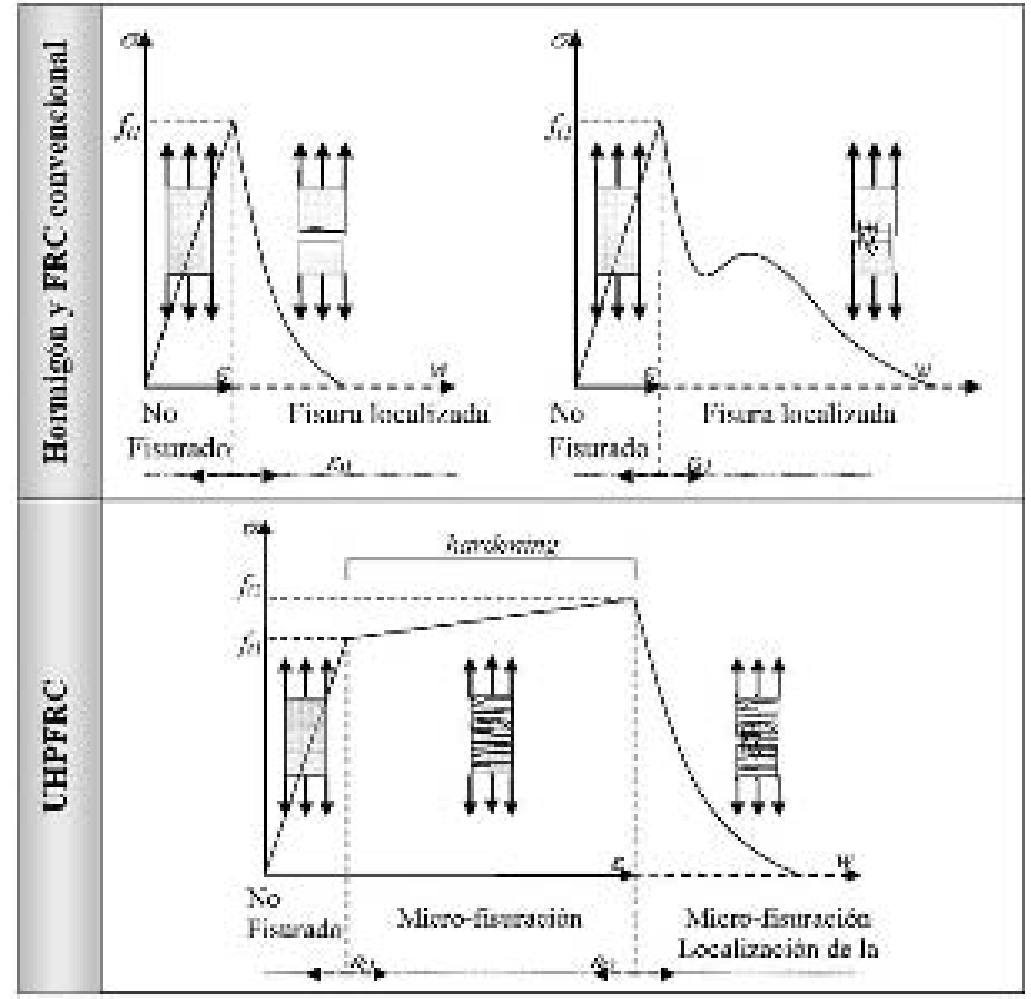

como las configuraciones de armado planteadas por Hua (8), Figura 2, o estrategias de confinamiento con el uso de laminas de materiales compuestos poliméricos, FRP, Figura 3 (9).

Una alternativa de simple ejecución para reducir la longitud de la zona de solape la constituye el aprovechamiento de la capacidad adherente de los UHPFRC, planteamiento estudiado por Aarup et al. (10) y Harryson (11) en el desarrollo de uniones para

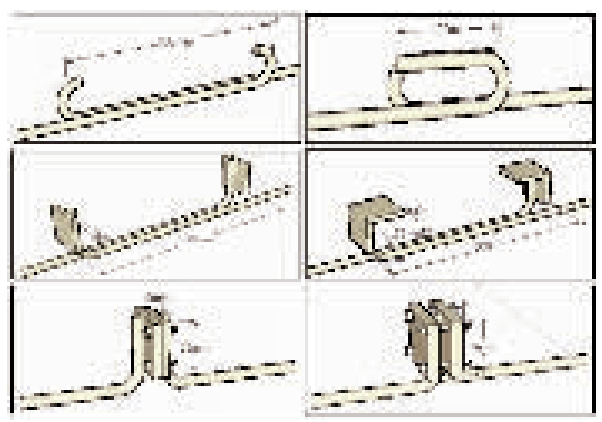

1. Comportamiento a tracción uniaxial de materiales de matriz de base cemento.

2. Dispositivos para solape de armaduras. Tomada de Hua (8).

3. Uso de FRP para el confinamiento de solapes, Hamad (9a). 
4. Esquema unión elementos de forjado con UHPFRC, CRC Tech-

5. Sección transversal elementos VT-1 y VT-2.

6. Descripción de las variables de las secciones consideradas. nology (12).

tableros de puentes, Figura 4, mediante el uso de barras rectas en lugar de las usuales conexiones con lazos, pero cuya aplicación en vigas ha sido referida en un numero limitado de ensayos (12).

\section{MOTIVACIÓN}

En el marco del trabajo de investigación para la puesta a punto de un sistema de conexión para pórticos prefabricados con el uso de UHPFRC, planteado a partir de las conclusiones del informe "Estudio para el uso de HPFRCC en conexiones de estructuras prefabricadas" presentado en el año 2007 (13), se estableció la necesidad de realizar una primera fase de evaluación del comportamiento de solapes de armaduras embebidos en UHPFRC en conexiones simples de vigas de hormigón armado. Los principales resultados y conclusiones de esta evaluación se presentan a continuación.

\section{PROGRAMA EXPERIMENTAL}

\subsection{Elementos y procedimiento}

El programa experimental se compuso de ensayos a flexión en cuatro puntos. En una primera fase se ensayaron tres elementos viga denominados Viga Tipo 1 (VT-1) y, en una segunda, se experimentó un único elemento denominado Viga Tipo 2 (VT-2). Todos los elementos vigas se construyeron a partir de medias vigas prefabricadas, de las cuales sobresalía, en una de sus caras, el refuerzo longitudinal, que se solapaba con el refuerzo saliente de la otra media viga en una zona central posteriormente hormigonada con un material del tipo UHPFRC, comercializado por CRC Technology.

Medias vigas con una sección transversal de $160 \mathrm{~mm}$ de ancho y $300 \mathrm{~mm}$ de canto y una longitud de 1,50 m configuraban los elementos VT-1. El refuerzo longitudinal en la cara superior e inferior estaba conformado por dos barras de diámetro $20 \mathrm{~mm}$. El refuerzo trasversal consistió de cercos cerrados de diámetro $8 \mathrm{~mm}$ espaciados $100 \mathrm{~mm}$, centro
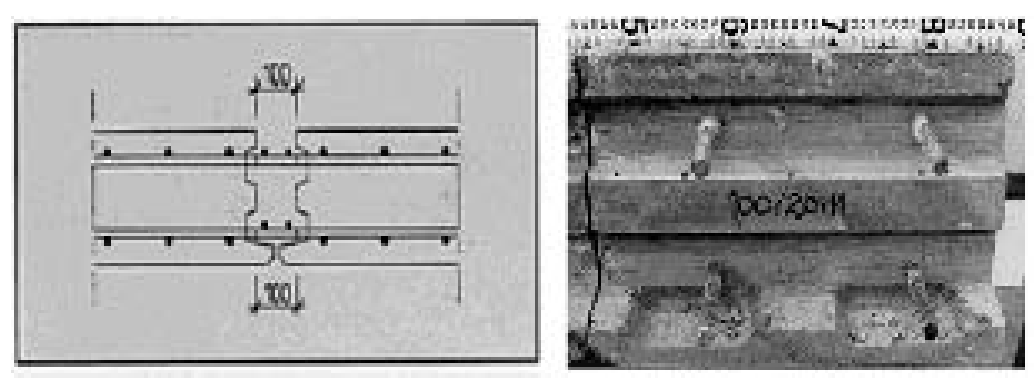

a centro, en la longitud de las medias vigas prefabricadas, pero sólo se dispusieron barras trasversales horizontales, sin los tirantes verticales, sobre y debajo del refuerzo longitudinal y espaciadas $100 \mathrm{~mm}$ en la zona de solape hormigonada con UHPFRC. Las longitudes de solape consideradas fueron $10 \mathrm{db}$ (VT-1A), 15db (VT-1B) y $20 \mathrm{db}$ (VT-1C). Con el objeto de permitir el solape de la armadura proveniente de las dos medias vigas a la altura de un mismo plano horizontal, el refuerzo no era simétrico con respecto al eje vertical que pasa por el centro de la sección. Por su parte, el elemento VT-2 estaba configurado por dos medias vigas de sección transversal, de $280 \mathrm{~mm}$ de ancho y $400 \mathrm{~mm}$ de canto, con una longitud de 2,0 m. El refuerzo longitudinal en la cara superior e inferior estaba conformado por tres barras de diámetro 20 $\mathrm{mm}$ y el refuerzo trasversal seguía el mismo esquema utilizado para los elementos VT-1. La longitud de solape considerada fue de $14 \mathrm{db}$. Detalles de la sección y el armado de las secciones se observan en la Figura 5.

Todos los elementos prefabricados se hicieron con hormigón autocompactable de 40 MPa en las instalaciones de la empresa Altresa del grupo PRAINSA en Aranda de Duero. El acero de refuerzo utilizado fue del tipo B 500SD, que satisfacía los requerimientos de la norma UNE 36065:2000 EX. (14)

Por su parte, Compact Reinforced Concrete (CRC), material del tipo UHPFRC comercializado por CRC Technology, fue utilizado para hormigonar las zonas de solape. El CRC se caracteriza por la utilización de polvos reactivos, comunes en los UHPFRC, y un contenido de fibras de acero, de $12 \mathrm{~mm}$ de longitud y $0,4 \mathrm{~mm}$ de diámetro, del 6\% en volumen (12). El material se fabricó siguiendo las principales indicaciones dadas por el fabricante, utilizando una hormigonera y los demás medios comunes en los emplazamientos de construcción, pero sin el uso de vibrador o ningún medio de compactación durante el vertido. La resistencia a compresión y flexo-tracción del material, Tabla 2, estuvo por debajo de las resistencias esperadas, sin embargo, el uso de una mayor energía durante el mezclado, mediante mezcladoras de eje vertical con palas, y medios de compactación simples pero adecuados, mesa vibradora para las muestras y vibradores de aguja para las zonas de conexión, ha demostrado su efectividad para alcanzar mayores resistencias en el material usado en el desarrollo de otras investigaciones actualmente en curso.

Las principales características variables de los ensayos y las propiedades de los materiales utilizados se presentan en las Tablas 1 y 2 . El valor cmin es el mínimo valor entre el recubrimiento inferior cb o lateral cs del refuerzo 
y la distancia media entre solapes s, Figura 6. Por su parte $f_{C}$ es la resistencia a compresión del hormigón determinada en probetas cilíndricas de $150 \mathrm{~mm}$ de diámetro, fCC es la resistencia a compresión del UHPFRC determinada en probetas cúbicas de $100 \mathrm{~mm}$ de lado y $f_{C} f$ es la resistencia a flexión determinada en probetas de $40 \times 40 \times 160 \mathrm{~mm}$

La carga se aplicó por medio de un gato hidráulico de 160 kN para los elementos VT-1 y de $600 \mathrm{kN}$ de capacidad para el elemento VT-2, siendo repartida en dos puntos de carga en los tercios de la luz mediante un perfil metálico, de tal forma que el momento en el tercio central, zona del solape, era constante, Figura 7, pág. 34. En la viga VT-1A, al ser $100 \mathrm{~mm}$ más corta se conservaron los puntos de aplicación de carga a una distancia de $1.040 \mathrm{~mm}$ desde cada uno de los apoyos, de tal forma que el momento en la zona central se corresponde con el aplicado en las vigas VT-1B y VT-1C

El proceso de carga se realiza mediante un proceso de carga escalonada con descarga completa, situación más desfavorable en virtud de la evidencia de una mayor degradación de la adherencia debido a la acción de cargas repetidas (15). La metodología planteada se inspira en el ensayo con variación de carga escalonada en sección bajo carril realizado para traviesas de hormigón y recogido en la norma UNE 13230:2003 (16). Se aplica inicialmente una rampa ascendente de carga hasta alcanzar el nivel de carga deseado, manteniéndola por un máximo de 5 minutos para realizar lecturas en la instrumentación dispuesta y se procede a la descarga, donde se realiza una nueva toma de lecturas
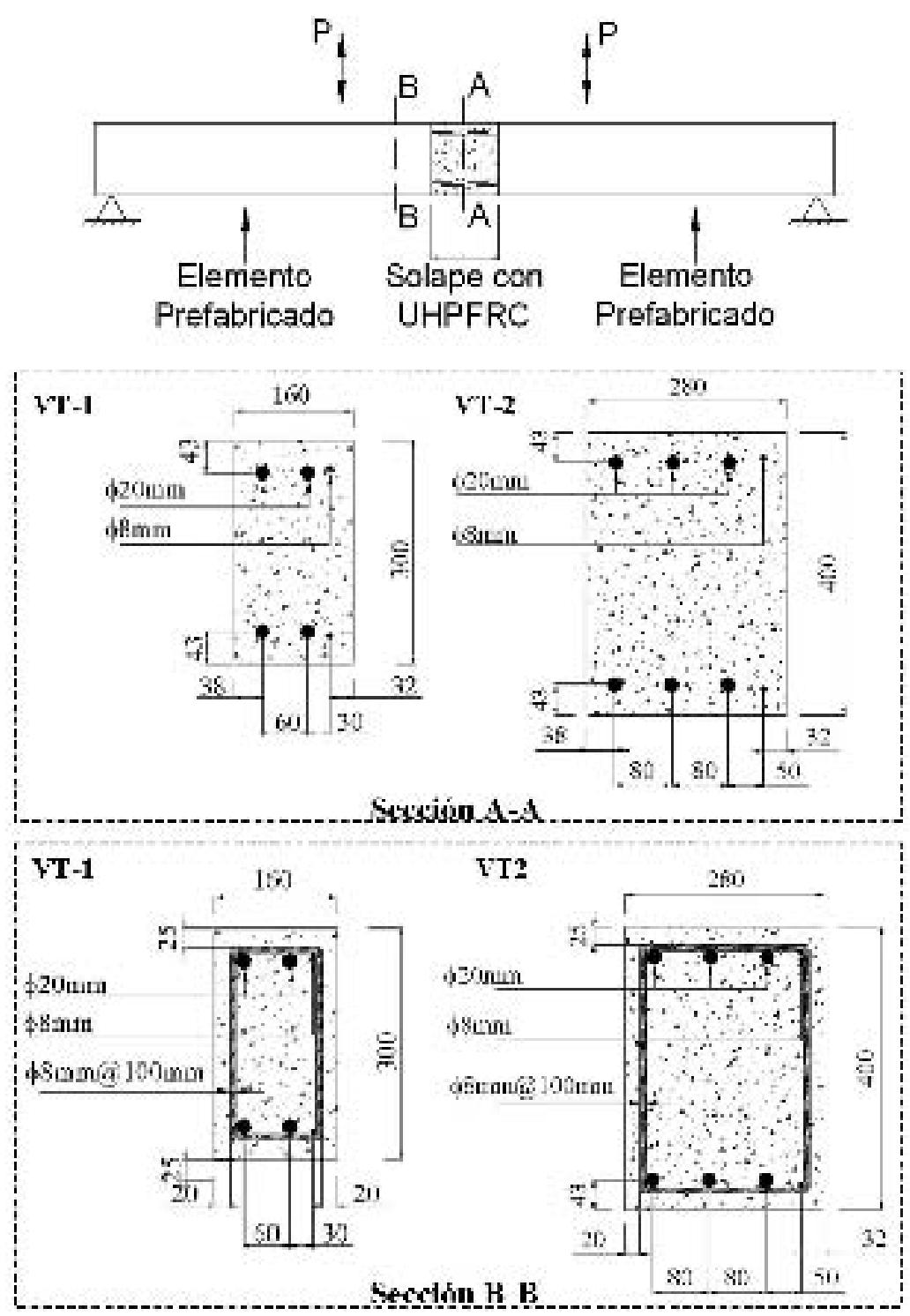

Tabla 1

Descripción de elementos para ensayos a flexión en cuatro puntos

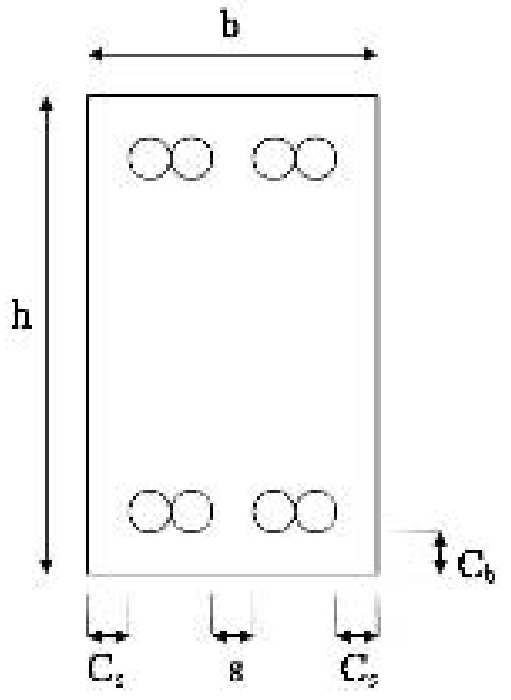

\begin{tabular}{|l|c|c|c|c|c|c|}
\hline \multicolumn{1}{|c|}{ Elemento } & $\begin{array}{c}\mathbf{C}_{\mathbf{m i ́ n}} \\
(\mathbf{m m})\end{array}$ & $\mathbf{c} / \mathbf{d}_{\mathbf{b}}$ & $\begin{array}{c}\mathbf{L}_{\text {solape }} \\
\mathbf{( \mathbf { m m } )}\end{array}$ & $\frac{L_{\text {solape }}}{d_{b}}$ & $\begin{array}{c}\mathbf{L}_{\text {UHPFRC }} \\
(\mathbf{m m})\end{array}$ & $\begin{array}{c}\mathbf{L}_{\text {libre }} \\
(\mathbf{m m})\end{array}$ \\
\hline VT-1A & 12 & 0,6 & 200 & 10 & 220 & 3.120 \\
\hline VT-1B & 12 & 0,6 & 300 & 15 & 320 & 3.120 \\
\hline VT-1C & 12 & 0,6 & 400 & 20 & 420 & 3.020 \\
\hline VT-2 & 17,3 & 0,86 & 280 & 14 & 300 & 4.000 \\
\hline
\end{tabular}

Tabla 2

Propiedades del hormigón y el UHPFRC

\begin{tabular}{|l|c|c|c|c|c|}
\hline \multirow{2}{*}{ Elemento } & \multicolumn{2}{|c|}{ Semiviga } & \multicolumn{3}{c|}{ Solape UHPFRC } \\
\cline { 2 - 6 } & $\begin{array}{c}f_{c} \\
(\mathbf{M P a})\end{array}$ & $\begin{array}{c}\text { Edad } \\
(\mathbf{d i ́ a s})\end{array}$ & $\begin{array}{c}f_{c c} \\
(\mathbf{M P a})\end{array}$ & $\begin{array}{c}f_{c f} \\
(\mathbf{M P a})\end{array}$ & $\begin{array}{c}\text { Edad } \\
(\mathbf{d i ́ a s})\end{array}$ \\
\hline VT-1A & 55,7 & 70 & 120,7 & 17,1 & 49 \\
\hline VT-1B & 65,7 & 67 & 101,9 & 15,8 & 49 \\
\hline VT-1C & 59,4 & 56 & 119,0 & 16,5 & 37 \\
\hline VT-2 & 55,7 & 70 & 151,3 & - & 117 \\
\hline
\end{tabular}



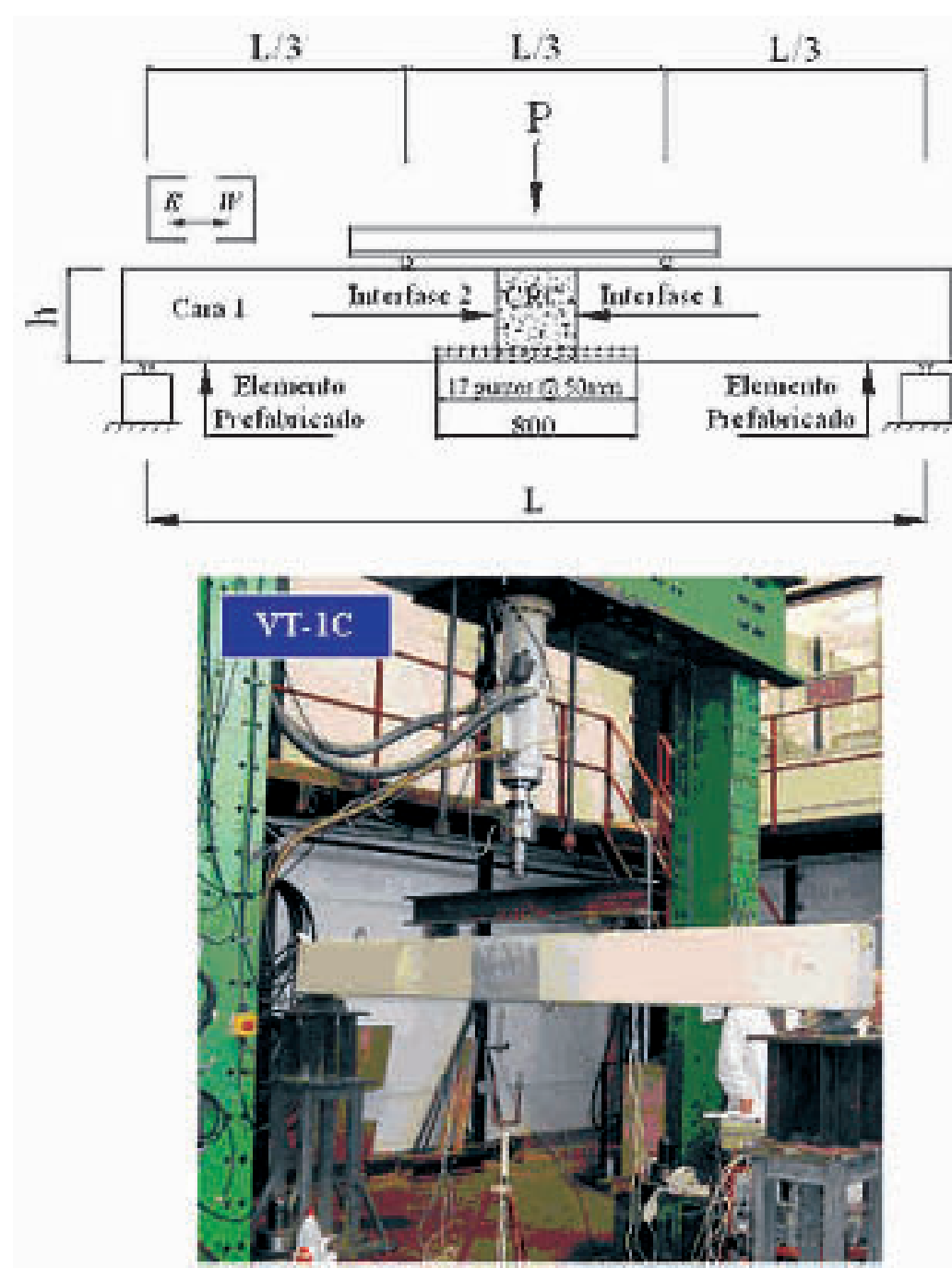

7. Montaje de ensayos a flexión en cuatro puntos.

8. Procedimiento de carga Elemento VT1

9. Disposición de las omegas y los puntos de medición.

10. Detalle localización de galgas extensométricas. Elementos VT1 y VT2.

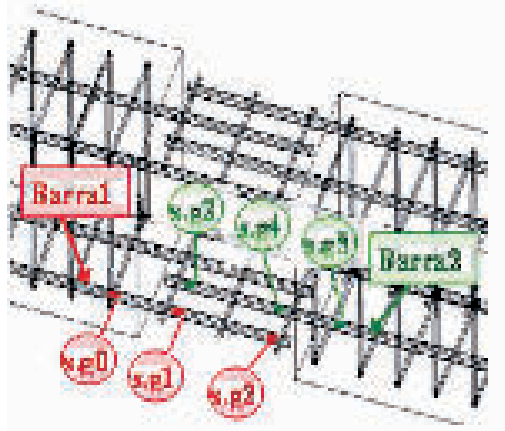

VI-1 en la instrumentación antes de proceder con

\subsection{Instrumentación}

Se obtuvo un completo registro de las cargas aplicadas de la célula de carga incorporada al gato utilizado, mientras las deflexiones en el punto central del elemento se registraron el siguiente paso de carga, Figura 8.
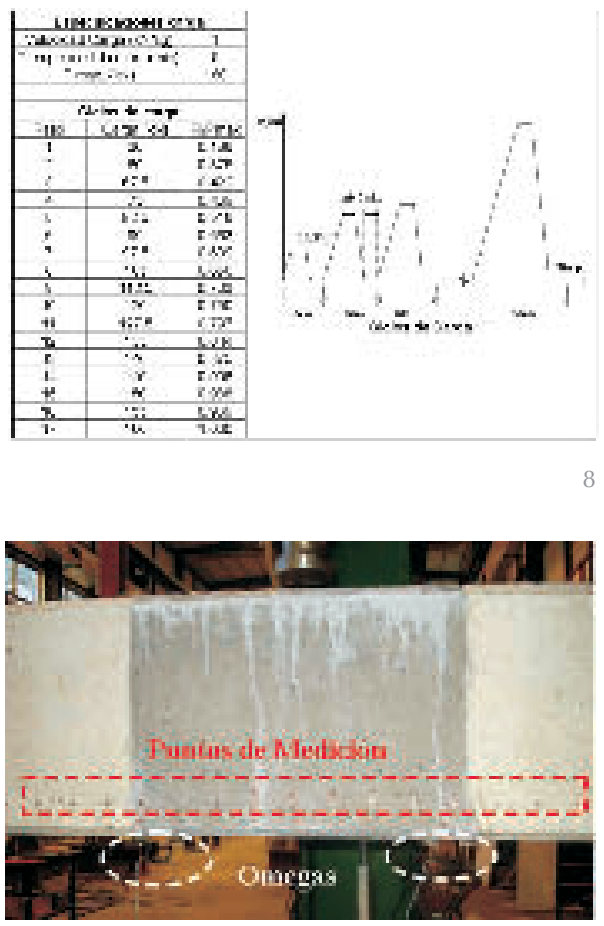

mediante una LVDT dispuesto en la cara inferior del elemento.

Se fijaron un total de 17 puntos de medición mecánica a la superficie del hormigón en ambas caras del elemento a la altura del refuerzo longitudinal inferior, espaciados aproximadamente $50 \mathrm{~mm}$, para la determinación de la elongación en la cuerda inferior en carga y descarga de cada uno de los ciclos. Adicionalmente se dispusieron omegas, elementos conformados por láminas metálicas con galgas extensométricas fijadas a su superficie, en las dos interfases formadas por el UHPFRC y el hormigón convencional, con el objeto de medir la apertura de la previsible fisura en esta ubicación. Con una amplitud máxima de $2 \mathrm{~mm}$ y una precisión de $0,01 \mathrm{~mm}$ las omegas se ubicaron en la cara inferior del elemento, Figura 9.

Se fijaron seis galgas extensométricas por cada elemento, tres en una barra inferior de cada una de las media vigas, para determinar las deformaciones en el refuerzo, Figura 10. En las vigas VT-1 la primera de ellas se localizó a $40 \mathrm{~mm}$ del extremo de la longitud sobresaliente de la barra, y las otras dos se ubicaron distanciadas $50 \mathrm{~mm}$ a cada lado del plano que correspondía a la interfase de los dos materiales. Esta disposición permitía que al posicionar las medias vigas para conformar el elemento, las galgas de una y otra media viga se correspondieran en la abscisa. En la viga VT-2 todas las galgas estaban dispuestas dentro de la región de UHPFRC. 


\section{RESULTADOS EXPERIMENTALES}

\subsection{Comportamiento general y modo de fallo}

En general todos los elementos presentaron un comportamiento similar previo a la rotura. Para los primeros ciclos de carga se hacían ya visibles las primeras fisuras a flexión en el hormigón convencional dentro de la zona de momento constante $y$, como se esperaba, se desarrollaban fisuras localizadas en la sección correspondiente a la interfase entre el hormigón convencional y el UHPFRC. Con el aumento de la carga se formaron fisuras adicionales, principalmente en el hormigón convencional por fuera de la zona de solape, y se incrementaba el ancho de la fisura en la secciones de la interfase entre los dos materiales. No es posible hablar de una fisuración localizada en el UHPFRC para niveles de carga en los que no se supera la capacidad a tracción postfisuración del material, Figura 1, sin embargo se observaron algunas fisuras en el UHPFRC para niveles medios de carga, valores de $80 \mathrm{kN}$ para los elementos VT-1 y 135 kN para el elemento VT-2 que corresponden a cerca de la mitad de la carga última esperada. Se trata de fisuras finas y de poca profundidad con separaciones cercanas a los $80 \mathrm{~mm}$, alcanzando con el aumento de la carga el nivel del refuerzo longitudinal inferior, Figura 11. Se formaron fisuras longitudinales, en dirección del refuerzo, a partir de las fisuras de flexión en las caras laterales y en las caras inferiores en el hormigón convencional de los elementos dentro de la zona de momento constante. Fisuras similares pero bastante finas se manifestaron posteriormente en la zona de UHPFRC $y$, finalmente, condicionaron el tipo de fallo observado en los elementos VT-1A y VT-1B.

En el elemento VT-1A la rotura se presentó por splitting del hormigón en la cara lateral, mientras que el elemento VT-1B desarrolló un fallo combinado a flexión y splitting en el cual el fallo por splitting en la cara lateral estuvo precedido por la plastificación del acero, Figura 12.

El elemento VT-1C se ensayó con el procedimiento descrito de ciclos de carga y descarga hasta agotar la capacidad del gato y posteriormente fue ensayado mediante control por desplazamiento hasta la rotura. La cedencia del acero, el agotamiento a compresión de la cabeza superior y una elevada flecha se observaron con anterioridad a la rotura del elemento, Figura 13.

Un comportamiento similar se observó en el elemento VT-2, con una longitud de solape de sólo $14 \mathrm{db}$, observándose el agotamiento de la cabeza a compresión en el hormigón convencional próximo a la interfase, la cedencia del acero y una gran deflexión previo a la rotura del elemento, Figura 14. Dentro

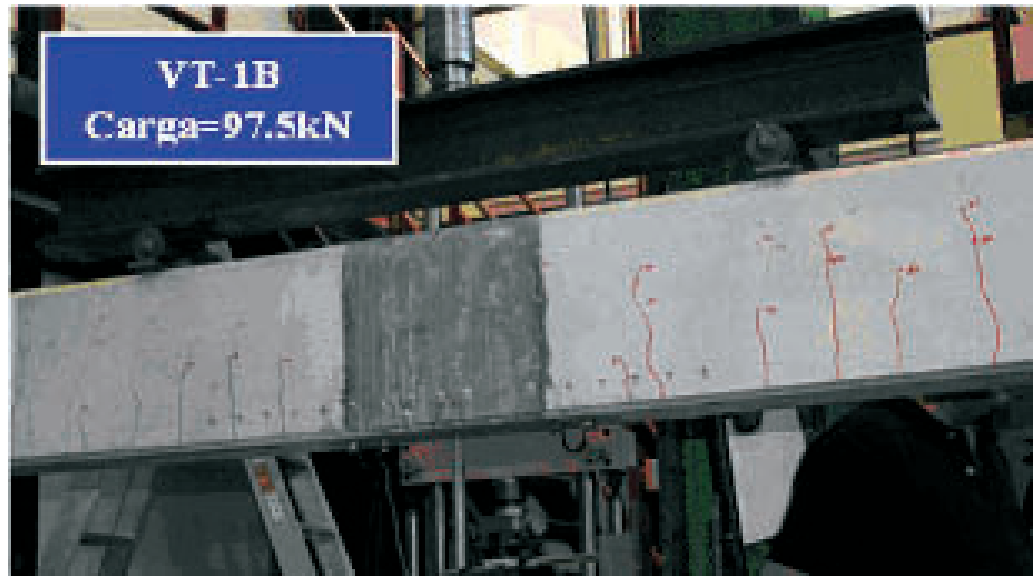

1
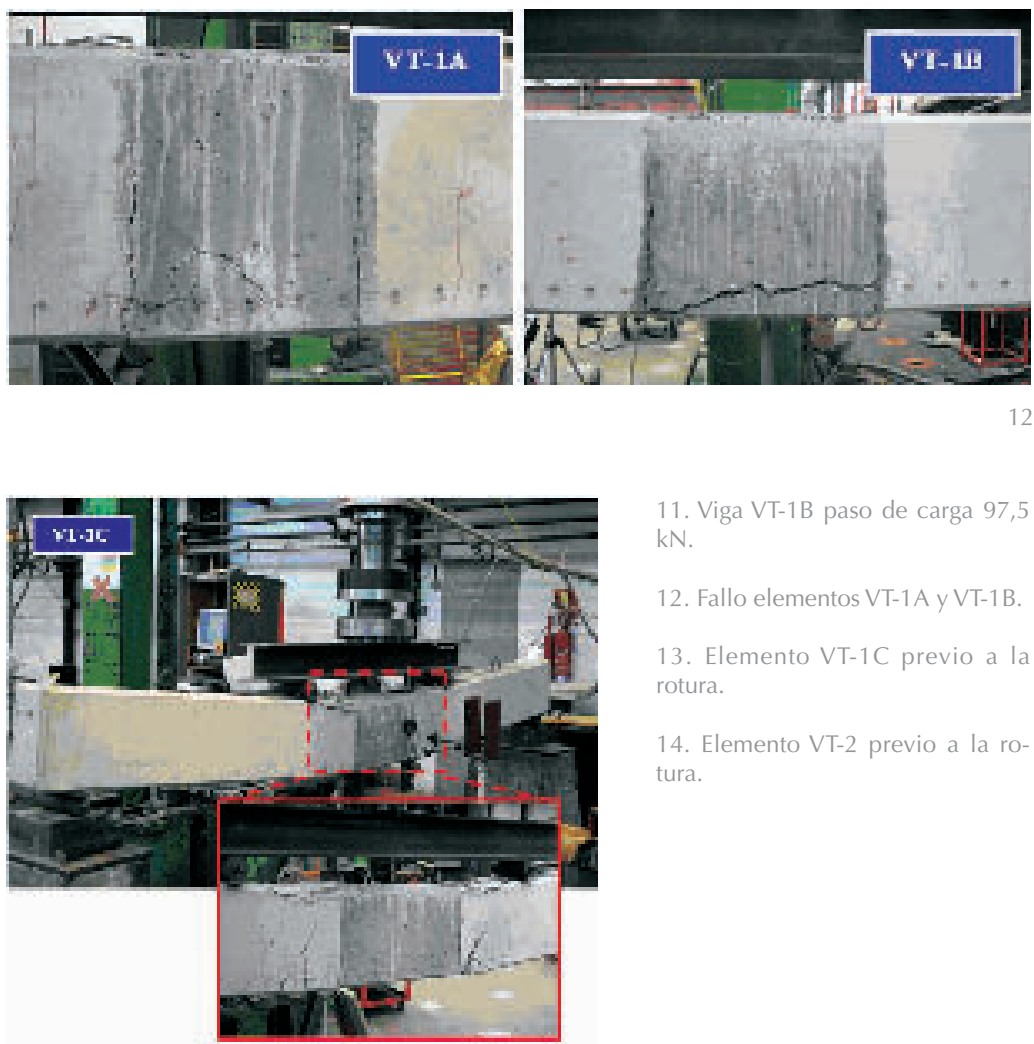

11. Viga VT-1B paso de carga 97,5 $\mathrm{kN}$

12. Fallo elementos VT-1A y VT-1B. 13. Elemento VT-1C previo a la rotura.

14. Elemento VT-2 previo a la rotura.

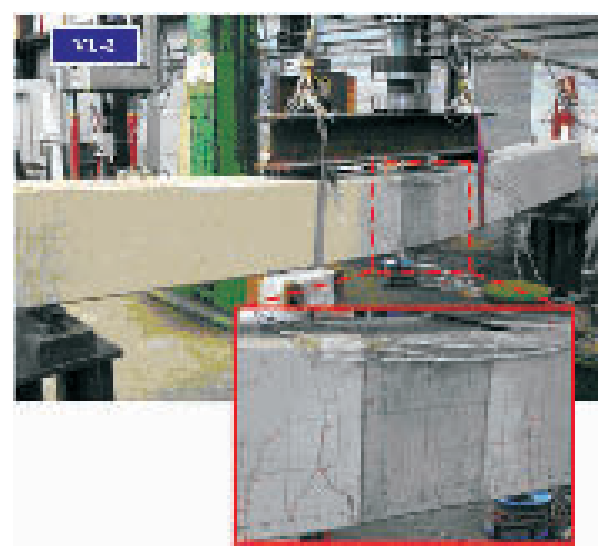



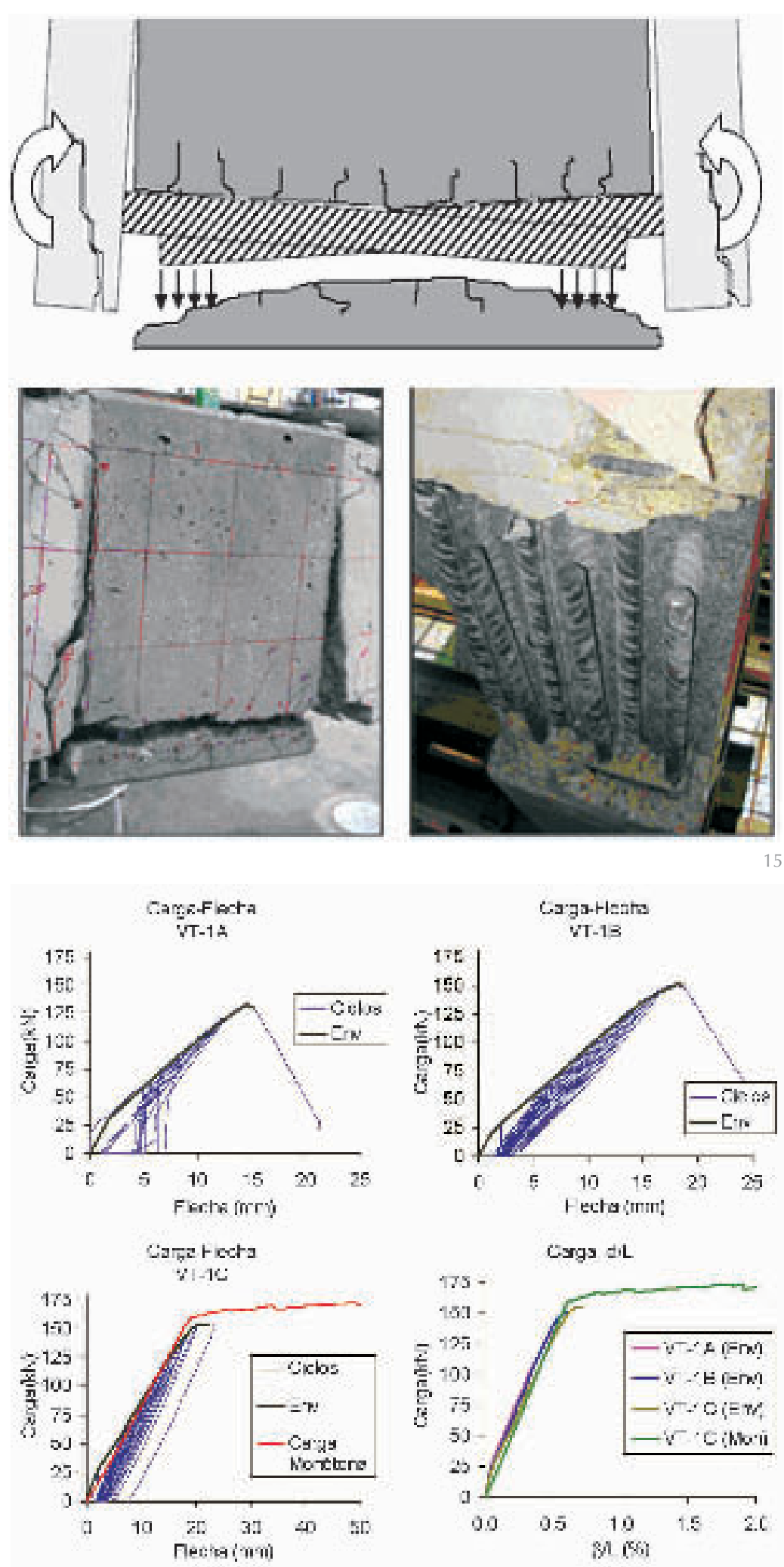

15. Esquema desprendimiento cuña inferior a causa de curvatura, fotografías elemento VT-2.

16. Curvas carga-flecha para los elementos VT-1.

17. Curvas carga-flecha para el elemento VT-2 de la zona de UHPFRC sólo se hicieron visibles unas pocas fisuras muy finas y de poca profundidad, siendo necesario destacar la mejor calidad del UHPFRC en relación al material de los elementos VT-1.

La rotura por la formación de una fisura longitudinal en la cara lateral de los elementos no fue condicionada por la capacidad de adherencia y resistencia al splitting del UHPFRC y se relaciona con el efecto de la curvatura observada en el elemento. En la configuración con gran deformación de la viga, para los cuales se mantenía aún la capacidad de carga, el empuje vertical que ejercen los extremos de las barras solapadas tiende a desprender la cuña inferior del material, efecto denominado prying (17), en un plano de fallo a la altura del refuerzo longitudinal, donde las tensiones resisten en su totalidad por el UHPFRC ante la ausencia de estribos cerrados o tirantes verticales y en un área efectiva bastante reducida dada la poca separación trasversal entre barras y solapes, Figura 15.

\subsection{Curvas carga-flecha}

La respuesta carga-flecha para los elementos ensayados se observa en las Figuras 16 y 17. Dado el procedimiento de ensayo, bajo control de carga con el fin de mantener aproximadamente el nivel de solicitación durante el periodo de tiempo en el que se tomaban los registros de los instrumentos de medición, no fue posible registrar el comportamiento posterior a la carga máxima en los elementos VT-1A y VT-1B que fallaron de forma frágil por splitting en la cara lateral. El elemento VT-1C, una vez sustituido el gato al superarse su capacidad de carga, y el elemento VT-2, después de ser superada la carga última esperada, se ensayaron hasta rotura mediante un proceso de carga por control de desplazamientos, siendo posible registrar una mayor ductilidad hasta la rotura.

Si bien se presentaron algunas dificultades con la lectura de la flecha durante la descarga en el elemento VT-1A, debido a inconvenientes con el flexímetro, se observa en general para todos los elementos el aumento de la flecha remanente a medida que trascurren los ciclos de carga y se produce la degradación de las propiedades de los materiales y los mecanismos de transferencias de carga, se originan deslizamientos relati-

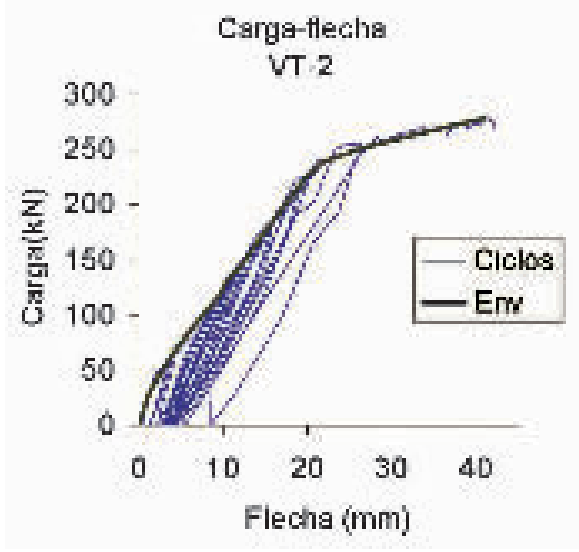


Tabla 3

Valores experimentales ensayos elementos VT-1 y VT-2

\begin{tabular}{|c|c|c|c|c|c|c|c|}
\hline Notación & $\begin{array}{l}\text { Carga } \\
\text { Máxima } \\
\text { P(kN) }\end{array}$ & $\begin{array}{l}\text { Deflexión } \\
\text { Máxima } \\
\delta(\mathrm{mm})\end{array}$ & $\begin{array}{c}\delta / L \\
\left(\times 10^{-3}\right)\end{array}$ & $\begin{array}{l}\text { Momento } \\
\text { máximo } \\
(\text { kN.m) }\end{array}$ & $\mathrm{M}_{\max } / \mathrm{M}_{\mathrm{u}}(\mathrm{c})$ & $\begin{array}{c}\boldsymbol{f}_{s} \\
(\mathrm{MPa})\end{array}$ & $\begin{array}{c}u_{s p} \\
(\mathrm{MPa})\end{array}$ \\
\hline VT-1A & 133,0 & 14,51 & 4,80 & 70,6 & 0,85 & 465,5 & 11,6 \\
\hline VT-1B & 150,6 & 18,06 & 5,79 & 79,8 & 0,96 & $516,0 a$ & 8,6 \\
\hline VT-1C & 174,1 & 56,60 & 18,14 & 92,0 & 1,11 & $516,0 \mathrm{a}$ & 6,5 \\
\hline VT-2 & 286,7 & $42,02^{(\mathrm{d})}$ & 10,51 & 196,7 & 1,07 & $516,0 \mathrm{a}$ & 9,2 \\
\hline \multicolumn{8}{|c|}{$\begin{array}{l}\text { Notas: a } \text { acero en cedencia } \\
{ }^{\mathrm{b}} M_{\text {máx }} \text { considerando el peso propio del elemento. } \\
{ }^{\mathrm{c}} M_{u} \text { determinado considerando un valor } f_{y} \text { de } 516 \mathrm{MPa} \text { y un factor de sobrerresistencia del acero de 1,25. } \\
{ }^{\mathrm{d}} \text { Se retiró el LVDT en un nivel de carga de } 270 \mathrm{kN}\end{array}$} \\
\hline
\end{tabular}

vos entre el hormigón y las barras de acero de refuerzo y las fisuración se extiende en el hormigón convencional. La comparación de la flecha relativa en los elementos VT-1 se presenta en la Figura 16, destacándose la ductilidad en el fallo del elemento VT-1C durante la segunda fase de ensayo bajo control de desplazamiento.

La Tabla 3 presenta la carga máxima $P_{\text {máx }}$ Y la correspondiente deflexión máxima $\delta_{\text {máx }}$ registrada, la tensión en el acero y la tensión de adherencia promedio en el fallo $u_{s p}$ para los diferentes elementos ensayados. La tensión $u_{s p}$ se determinó mediante la expresión [1], en función del diámetro y área de la barra de refuerzo, $A_{b}$ y $d_{b}$ respectivamente, la longitud del solape $L_{s / p}$ y la tensión en las barras de acero $f_{s}$ determinada mediante un análisis seccional en la sección de hormigón convencional en la zona de momento constante próxima al UHPFRC y verificada por la lectura de los extensómetros adheridos al refuerzo.

$$
u_{s p}=\frac{A_{b} f_{s}}{\pi d_{b} L_{s i p}}
$$

\subsection{Relaciones carga-deformación}

La Figura 18 presenta la variación de las deformaciones medidas en el acero durante los diferentes ciclos de carga. Se presentaron algunos inconvenientes en los registros obtenidos para el elemento VT-1A, donde una galga extensométrica en la barra 1 y dos galgas en la barra 2 no funcionaron adecuadamente, y en el elemento VT-2 donde una galga no registró.

Adicionalmente se presenta en la Figura 19, pág. siguiente, un registro de las deformaciones en el acero solapado a lo largo de un perfil de los elementos estudiados para diferentes niveles de carga a partir de los datos obtenidos de las bandas extensométricas adheridas al refuerzo.

Se registran para los elementos VT-1B, VT1C y VT-2 valores de deformación superiores
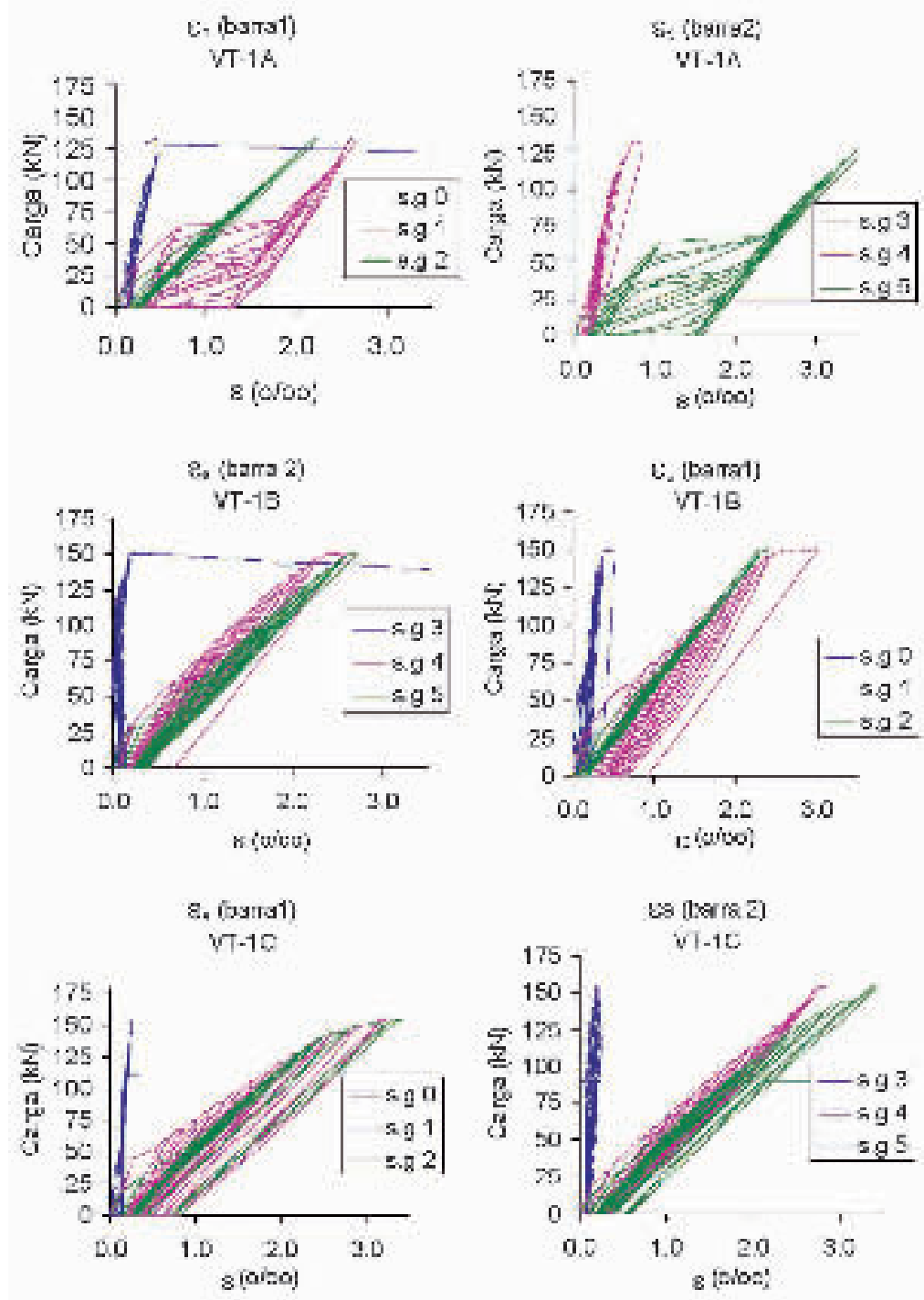

18. Relación carga-deformación en el acero de refuerzo. 

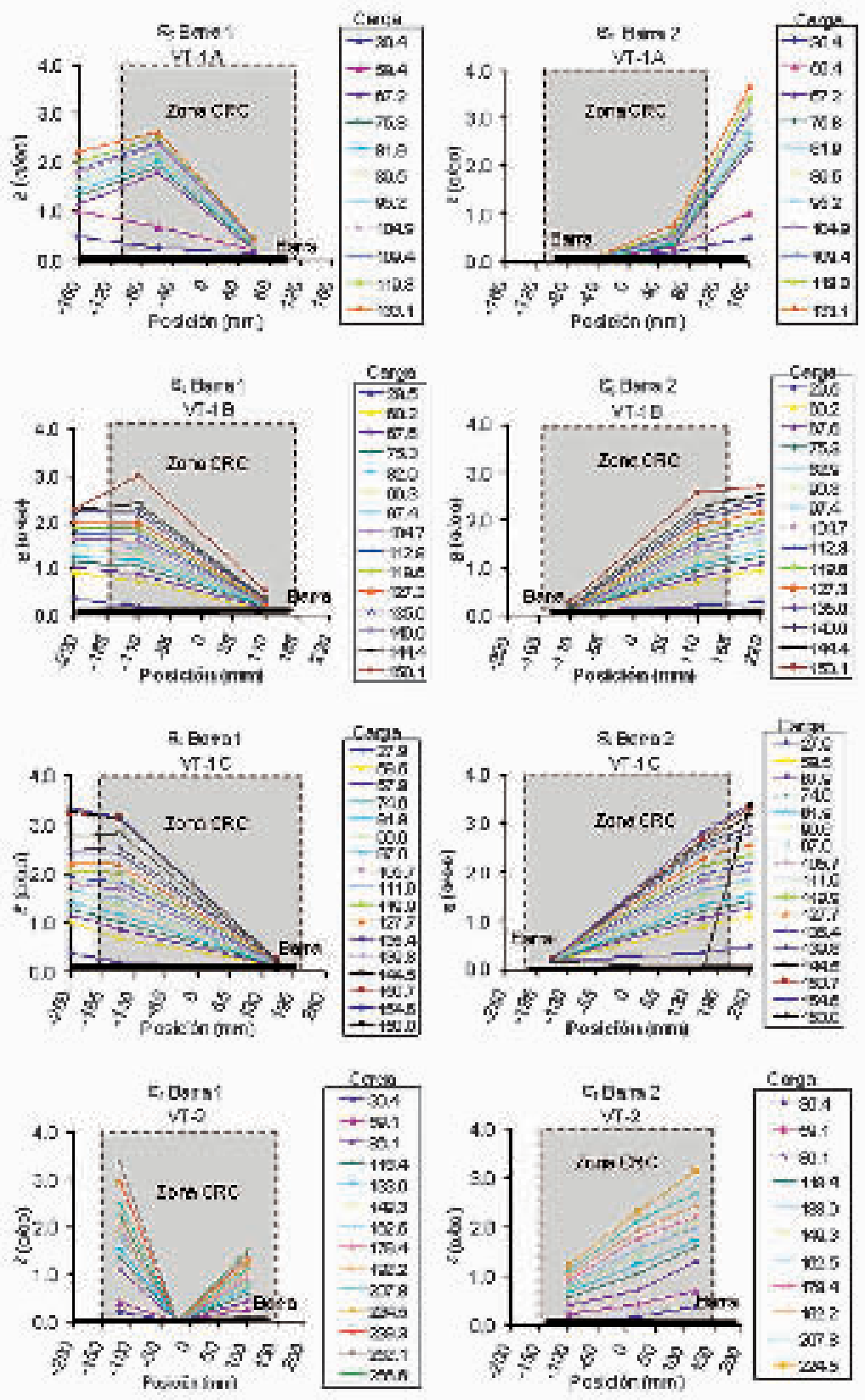

19. Perfil de deformaciones en la armadura solapada. En orden descendente, elementos VT-1A, VT-1B, VT-1C y VT-2. al límite elástico del acero a ambos lados de las interfases, es decir, tanto en la sección ubicada en el hormigón convencional como en el UHPFRC. Un completo desarroIlo de la capacidad resistente de las barras solapadas, en función de la definición de longitud de desarrollo, es alcanzado para estos dos elementos. Es evidente, dadas las cortas longitudes de solape utilizadas, la influencia del comportamiento a tracción del material, su capacidad y ductilidad, en los mecanismos de transferencia de tensiones y el efecto rigidizador del UHPFRC en el tirante de la cuerda inferior del elemento. Un estudio más detallado de las tensiones en la barra de refuerzo embebida que el llevado a cabo en este estudio, mediante el uso de un mayor número de galgas extensométricas, permitiría determinar de forma más precisa la magnitud de estos efectos. Por su parte, los niveles de deformación observados para la barra 1 del elemento VT-1A, ya que las bandas de la barra 2 no registraron adecuadamente, son también muy próximos a la plastificación, pero fue el recubrimiento lateral el factor determinante para el fallo por adherencia.

Se estableció un perfil de la deformación en la cara lateral de los elementos a la altura del refuerzo longitudinal a partir de las deformaciones medidas en los 17 puntos de medición dispuestos en la zona de momento constante, Figura 20.

En la posición de la fibra estudiada, sometida predominantemente a tracción, la distribución observada de las deformaciones en la zona de solape se corresponde con el comportamiento a tracción, la mayor rigidez y ductilidad de los UHPFRC, observándose una mayor uniformidad y una menor magnitud de las deformaciones en comparación con el comportamiento observado en el hormigón convencional, donde la concentración de las deformaciones en las zonas de formación de fisuras por flexión daba lugar al registro de picos con espaciamientos que se corresponde con el patrón de fisuración observado, fisuras discretas espaciadas aproximadamente $100 \mathrm{~mm}$.

Los perfiles de deformación registran, como se esperaba, la formación y el crecimiento de la fisura en o cerca de la interfase de los dos materiales. Fue a partir de las interfases, las cuales acusaron la mayor parte del daño como se verifica en los registros de deformaciones remantes tras las descargas y en las omegas de medida en la Figura 21, que se comenzó a observar la formación de las fisuras por splitting tanto en la cara lateral como en la cara inferior de los elementos VT-1A y VT-1B.

El comportamiento del solape bajo las condiciones de ensayo, y para longitudes cortas de solape como las utilizadas, es adecuado, y la transferencia de fuerzas, efectiva.

Las fisuras en la interfase responden a la configuración del ensayo, donde la pérdida inicial de la adherencia ocurrida en el hormigón convencional en las proximidades de la interfase entre los materiales resulta en la concentración de tensiones y la discontinuidad en las deformaciones entre el refuerzo y la matriz.

El comportamiento del tirante a tracción es gobernado por el anclaje de la longitud de barra embebida en la región de UHPFRC donde se produce el solape, todo ello bajo un proceso de carga repetida, que si bien 
no presenta inversión de sentido, si alcanza prácticamente la descarga, situación desfavorable y que ocasiona una mayor degradación de la ley de adherencia entre el acero y la matriz (15). Con el aumento de la carga se amplía la zona con perdida de adherencia en el elemento de hormigón convencional y se inicia la penetración de la deformación en el tirante anclado en UHPFRC que, finalmente, compromete la resistencia del mismo, como se observó en el elemento VT-1A, Figura 20.

\section{EVALUACIÓN DEL USO DE UHPFRC}

El desarrollo de la capacidad última en los elementos con longitud de solape de $14 \mathrm{~d}_{b}$ sin contar con el uso de cercos cerrados o tirantes verticales de refuerzo trasversal, pone de manifiesto la relevancia de las propiedades del UHPFRC utilizado en el desarrollo de conexiones por adherencia con longitudes de solape corta. A pesar del poco recubrimiento lateral y la ajustada separación trasversal entre solapes, solamente el elemento VT-1A presentó un fallo típico de adherencia debido al splitting lateral, a un nivel de carga cercano al $85 \%$ de la carga última. En los demás elementos la plastificación del acero y el aumento de la flecha condicionaron la rotura, en un modo combinado flexiónsplitting para el elemento VT-1B y mediante el desprendimiento derivado de la curvatura para los elementos VT-1C y VT-2.

Las investigaciones sobre el comportamiento de los solapes de armaduras en hormigón convencional son abundantes $y$ el uso de FRC y confinamiento mediante FRP como medio para mejorar su desempeño han sido evaluados por diversos autores. Como se refirió con anterioridad la información disponible sobre el uso de UHPFRC en solapes cortos de armadura es escasa, pero se desprende de los resultados obtenidos en el desarrollo de esta investigación y los reportados por Aarup et al. (10) y Harryson (11) la efectividad del uso de este tipo de materiales para reducir las longitudes de anclaje sin perjudicar la capacidad del elemento en relación a otros medios estudiados.

La Tabla 4 (pág. siguiente) y la Figura 21, pág. 41, permiten establecer una valoración comparativa con elementos similares a los utilizados en esta investigación fabricados con hormigones con y sin fibras, siendo $V f$ el volumen de fibra en los FRC. Los elementos referidos corresponden a vigas ensayadas a flexión en cuatro puntos con el solape localizado en la región de momento constante y cuya armadura de refuerzo estaba constituida por barras de diámetro $20 \mathrm{~mm}$.
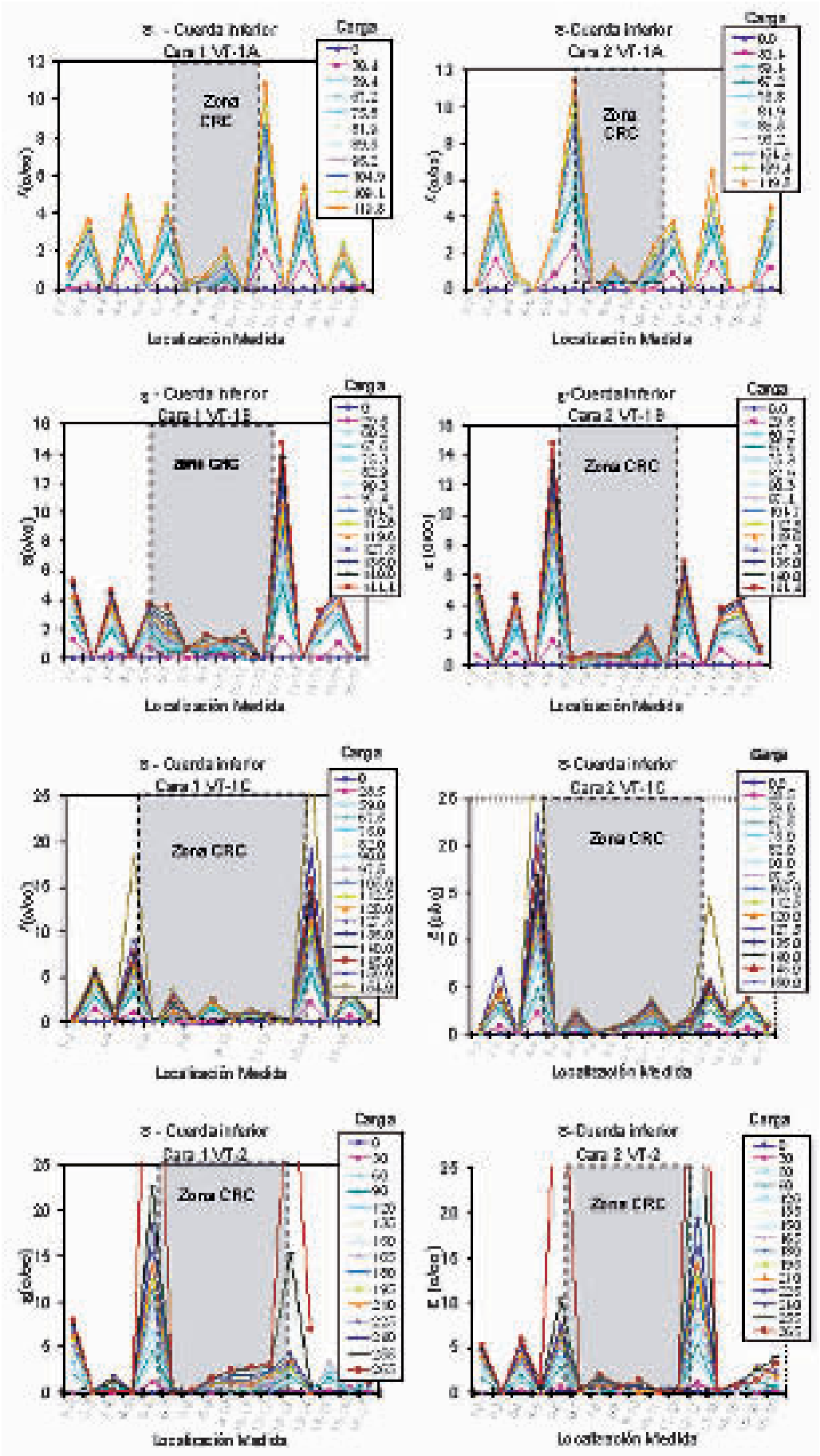

En todos ellos, a excepción de los elementos ensayados por Harajli y Gharzeddine (18), se utilizó un proceso de carga monótona, condición menos exigente que la utilizada en el desarrollo de este estudio. Todos los elementos referidos presentaron fallo por splitting a excepción de los elementos NB20F1.0 y NB20F1.5 con fallos combinados flexión-splitting.

Con unas longitudes de solape de tan sólo $15 \mathrm{db}$, la capacidad de los elementos se ve condicionada por el splitting como mecanismo de fallo dominante en las secciones 
Tabla 4

Resultados experimentales de referencia de ensayos a flexión en solapes

\begin{tabular}{|c|c|c|c|c|c|c|c|c|c|c|c|c|}
\hline Notación & $\begin{array}{c}\mathrm{fc} \\
(\mathrm{MPa})\end{array}$ & $\begin{array}{c}f y \\
(\mathrm{MPa})\end{array}$ & $\begin{array}{l}\mathrm{Vf} \\
(\%)\end{array}$ & $c / d_{b}$ & $\frac{L_{\text {solape }}}{d_{b}}$ & $\begin{array}{c}\text { Carga } \\
\text { Máxima } \\
\mathbf{P}(\mathbf{k N})\end{array}$ & $\begin{array}{c}\text { De- } \\
\text { flexión } \\
\text { Máxima } \\
\delta(\mathrm{mm})\end{array}$ & $\begin{array}{c}\delta / L \\
\left(10^{-3}\right)\end{array}$ & $\begin{array}{c}\text { Mo- } \\
\text { mento } \\
\text { máximo } \\
\text { b }(k N . m)\end{array}$ & $\frac{M_{m a x}}{M_{u}{ }^{(c)}}$ & $\mathrm{f}_{\mathrm{s}}(\mathrm{MPa})$ & $\begin{array}{c}\mathbf{u}_{\text {split }} \\
(\mathrm{MPa})\end{array}$ \\
\hline \multicolumn{13}{|c|}{ Harajli y Salloukh (4) } \\
\hline B-6-6-C & 44,3 & 517 & - & 2,1 & 15,8 & 152,3 & 4,13 & 2,06 & 53,9 & 0,66 & 348,2 & 5,5 \\
\hline B7-6-S-60-2 & 42,7 & 517 & 2,0 & 2,1 & 15,8 & 219,8 & 7,61 & 3,80 & 77,4 & 0,95 & 500,6 & 7,9 \\
\hline B8-6-S-60-1.2 & 43,0 & 517 & 1,2 & 2,1 & 15,8 & 206,6 & 6,99 & 3,49 & 72,8 & 0,89 & 472,3 & 7,5 \\
\hline B9-6-S-100-0.45 & 31,2 & 517 & 0,5 & 2,1 & 15,8 & 153,2 & 3,94 & 1,97 & 54,2 & 0,68 & 348,2 & 5,5 \\
\hline B10-6-S-100-0.6 & 37,2 & 517 & 0,6 & 2,1 & 15,8 & 170,5 & 4,84 & 2,42 & 60,2 & 0,75 & 388,2 & 6,1 \\
\hline \multicolumn{13}{|c|}{ Harajli y Gharzeddine (18) } \\
\hline NB20F0.0 & 42,8 & 508 & - & 2,0 & 20,0 & 176,0 & - & - & 62,4 & 0,79 & 434,0 & 5,4 \\
\hline NB20F0.5 & 42,8 & 508 & 0,5 & 2,0 & 20,0 & 220,0 & - & - & 77,8 & 0,99 & 512,0 & 6,4 \\
\hline NB20F1.0 & 42,8 & 508 & 1,0 & 2,0 & 20,0 & 227,0 & - & - & 80,2 & 1,02 & $512,0^{\text {a }}$ & 6,4 \\
\hline NB20F1.5 & 42,8 & 508 & 1,5 & 2,0 & 20,0 & 228,0 & - & - & 80,6 & 1,03 & $515,0^{\text {a }}$ & 6,4 \\
\hline \multicolumn{13}{|l|}{ Turk et al. (19) } \\
\hline B.NC.20 & 41,5 & 510 & - & 1,5 & 15,5 & 188,2 & 5,27 & 2,64 & 57,1 & 0,68 & 358,2 & 5,8 \\
\hline B.SCC. 20 & 44,1 & 510 & - & 1,5 & 15,5 & 202,0 & 5,45 & 2,73 & 60,6 & 0,72 & 384,2 & 6,2 \\
\hline \multicolumn{13}{|l|}{ Harajli et al. (20) } \\
\hline B2NF0 & 27,5 & 448 & - & 0,88 & 5,0 & 40,2 & 1,10 & 1,00 & 8,1 & 0,17 & 87,6 & 4,4 \\
\hline B2NF0.5 & 32,1 & 448 & 0,5 & 0,88 & 5,0 & 46,1 & 1,24 & 1,13 & 9,3 & 0,19 & 100,4 & 5,0 \\
\hline B2NF1.0 & 25,3 & 448 & 1,0 & 0,88 & 5,0 & 47,9 & 1,43 & 1,30 & 9,7 & 0,20 & 104,4 & 5,2 \\
\hline B2NF2.0 & 28,7 & 448 & 2,0 & 0,88 & 5,0 & 51,6 & 1,65 & 1,50 & 10,4 & 0,22 & 112,5 & 5,6 \\
\hline B2WF0 & 27,5 & 448 & - & 1,50 & 5,0 & 49,0 & 1,07 & 0,97 & 9,9 & 0,20 & 113,7 & 5,7 \\
\hline B2WF0.5 & 32,1 & 448 & 0,5 & 1,50 & 5,0 & 57,5 & 1,50 & 1,36 & 11,6 & 0,24 & 132,1 & 6,6 \\
\hline B2WF1.0 & 25,3 & 448 & 1,0 & 1,50 & 5,0 & 60,9 & 2,54 & 2,31 & 12,3 & 0,25 & 141,2 & 7,1 \\
\hline B2WF2.0 & 28,7 & 448 & 2,0 & 1,50 & 5,0 & 64,8 & 2,05 & 1,86 & 13,1 & 0,27 & 150,2 & 7,5 \\
\hline \multicolumn{13}{|l|}{ Hamad et al. (21) } \\
\hline B20F0 & 64,0 & 639 & - & 1,0 & 15,3 & 260,0 & 5,89 & 2,95 & 91,5 & 0,52 & 326,9 & 5,4 \\
\hline B20F0.5 & 68,0 & 639 & 0,5 & 1,0 & 15,3 & 290,6 & 10,60 & 5,30 & 102,1 & 0,58 & 364,1 & 6,0 \\
\hline B20F1.0 & 77,6 & 639 & 1,0 & 1,0 & 15,3 & 357,6 & 16,00 & 8,00 & 125,5 & 0,71 & 446,2 & 7,3 \\
\hline B20F2.0 & 60,3 & 639 & 2,0 & 1,0 & 15,3 & 391,2 & 17,98 & 8,99 & 137,2 & 0,78 & 490,5 & 8,0 \\
\hline \multicolumn{13}{|l|}{ Hamad et al. (9) } \\
\hline No & 27,5 & 592 & - & 1,00 & 15,3 & 174,0 & 3,81 & 1,91 & 61,5 & 0,38 & 184,9 & 3,03 \\
\hline NC1S1 & 28,4 & 592 & - & 1,00 & 15,3 & 195,0 & 4,18 & 2,09 & 68,8 & 0,42 & 207,1 & 3,39 \\
\hline NC1S2 & 29,8 & 592 & - & 1,00 & 15,3 & 200,8 & 4,45 & 2,23 & 70,8 & 0,43 & 213,0 & 3,49 \\
\hline NC1S3 & 31,1 & 592 & - & 1,00 & 15,3 & 226,6 & 4,83 & 2,42 & 79,8 & 0,49 & 240,4 & 3,94 \\
\hline NC2S1 & 35,8 & 592 & - & 1,00 & 15,3 & 214,6 & 4,06 & 2,03 & 75,6 & 0,46 & 227,7 & 3,73 \\
\hline NC2S2 & 28,4 & 592 & - & 1,00 & 15,3 & 209,2 & 4,19 & 2,10 & 73,7 & 0,45 & 221,9 & 3,64 \\
\hline NC2S3 & 29,2 & 592 & - & 1,00 & 15,3 & 237,6 & 5,84 & 2,92 & 83,6 & 0,51 & 251,8 & 4,13 \\
\hline \multicolumn{13}{|l|}{ Hamad et al. (9) } \\
\hline $\mathrm{BO}$ & 47,3 & 592 & & 1,00 & 15,3 & 302,6 & 4,40 & 2,20 & 106,3 & 0,65 & 357,6 & 5,86 \\
\hline BG1S1 & 58,9 & 592 & & 1,00 & 15,3 & 314,0 & 5,60 & 2,80 & 110,3 & 0,67 & 407,4 & 6,68 \\
\hline BG1S2 & 51,1 & 592 & & 1,00 & 15,3 & 349,0 & 6,10 & 3,05 & 122,5 & 0,75 & 452,4 & 7,42 \\
\hline BG1S3 & 52,3 & 592 & & 1,00 & 15,3 & 357,0 & 6,40 & 3,20 & 125,3 & 0,76 & 462,9 & 7,59 \\
\hline BG2S1 & 51,5 & 592 & & 1,00 & 15,3 & 312,6 & 5,10 & 2,55 & 109,8 & 0,67 & 405,4 & 6,65 \\
\hline BG2S2 & 49,8 & 592 & & 1,00 & 15,3 & 359,4 & 5,20 & 2,60 & 126,1 & 0,77 & 465,9 & 7,64 \\
\hline NG2S3 & 50,8 & 592 & & 1,00 & 15,3 & 373,6 & 7,00 & 3,50 & 131,1 & 0,80 & 484,2 & 7,94 \\
\hline BC1S1 & 63,2 & 592 & & 1,00 & 15,3 & 314,6 & 5,20 & 2,60 & 110,5 & 0,67 & 408,2 & 6,69 \\
\hline BC1S2 & 57,7 & 592 & & 1,00 & 15,3 & 333,4 & 6,00 & 3,00 & 117,1 & 0,71 & 432,5 & 7,09 \\
\hline BC1S3 & 55,2 & 592 & & 1,00 & 15,3 & 364,6 & 6,30 & 3,15 & 127,9 & 0,78 & 472,5 & 7,75 \\
\hline
\end{tabular}

de hormigón armado y FRC convencionales con los recubrimientos usuales, sin embargo con el UHPFRC utilizado se produjo la plastificación de la armadura para longitudes de $14 \mathrm{db}$, condición que sólo se presentó en los elementos NB20F1.0 y NB20F1.5 de los ensayados por Harajli y Gharzeddine (18), con longitud de solape de $20 \mathrm{db}$, y un factor de recubrimiento, $\mathrm{c} / \mathrm{db}$, superior a tres veces el utilizado en este estudio.

La capacidad última registrada para los elementos VT-1C y VT-2 superó en $11 \%$ y $7 \%$ la capacidad última calculada, Figura 22, pre- 
sentando una considerable deflexión antes de la rotura del elemento. En general, el uso del UHPFRC para la conformación de solapes de barras de refuerzo permite el desarrollo un mayor porcentaje de la capacidad a flexión de los elementos en relación al uso de hormigones y FRC convencionales, lo que se traduce en la necesidad de longitudes de solape más cortas incluso con el uso de menores recubrimientos. La Tabla 5 presenta las longitudes de solape requeridas por la Instrucción EHE (22), el Codigo Modelo-90 (1) y la norma ACI 31808 (23) para solapes bajo condiciones similares a las utilizadas en este estudio, solape de la totalidad del refuerzo en una misma sección, pero con recubrimientos y separaciones entre barras y solapes acordes a los requerimientos mínimos de cada instrucción. Las longitudes requeridas superan ampliamente las longitudes de solape entre $10 \mathrm{db}$ y $20 \mathrm{db}$ adoptadas en esta investigación.

Resulta ampliamente reconocida la mejora en las condiciones de confinamiento y adherencia con el uso de los FRC, como se verifica en los resultados experimentales reportados por Harajli y Salloukh (4) y Harajli y Gharzeddine (18). Sin embargo, el uso de UHPFRC permite hacer efectivo dicho confinamiento con recubrimientos o separaciones trasversales entre solapes ajustadas, como los que se pueden derivar de las limitaciones en las dimensiones de los elementos y la congestión en la armadura de refuerzo en elementos prefabricados. Para los cuatro elementos ensayados la separación entre los solapes era bastante limitada, con valores de $\mathrm{c} / \mathrm{db}$, tan bajos como 0,6, pero presentando un comportamiento adherente satisfactorio.

La cedencia de las barras de refuerzo para longitudes de anclaje superiores a $14 \mathrm{db}$ en los solapes de armaduras conformados con el UHPFRC utilizado, hace necesario plantear el uso de solapes aún más cortos para evaluar detalladamente la distribución y el valor de las tensiones de adherencia mediante ensayos en vigas, dada la modificación en las condiciones de adherencia debidas a la plastificación del acero y la concentración de las deformaciones. El resultado observado para el elemento VT-1A permite sin embargo evidenciar un aumento en el valor de la tensión de adherencia promedio en el fallo por splitting, usp, en relación a otros elementos con mayores recubrimientos, ver Tabla 4.

El uso de UHPFRC, y en particular el material CRC usado en este estudio, constituyen una alternativa viable para mejorar el comportamiento de conexiones en elementos de hormigón armado donde se no se disponga de las longitudes suficientes para el solape de la armadura, con algunas ventajas sobre alternativas como la planteada por Hua (8),
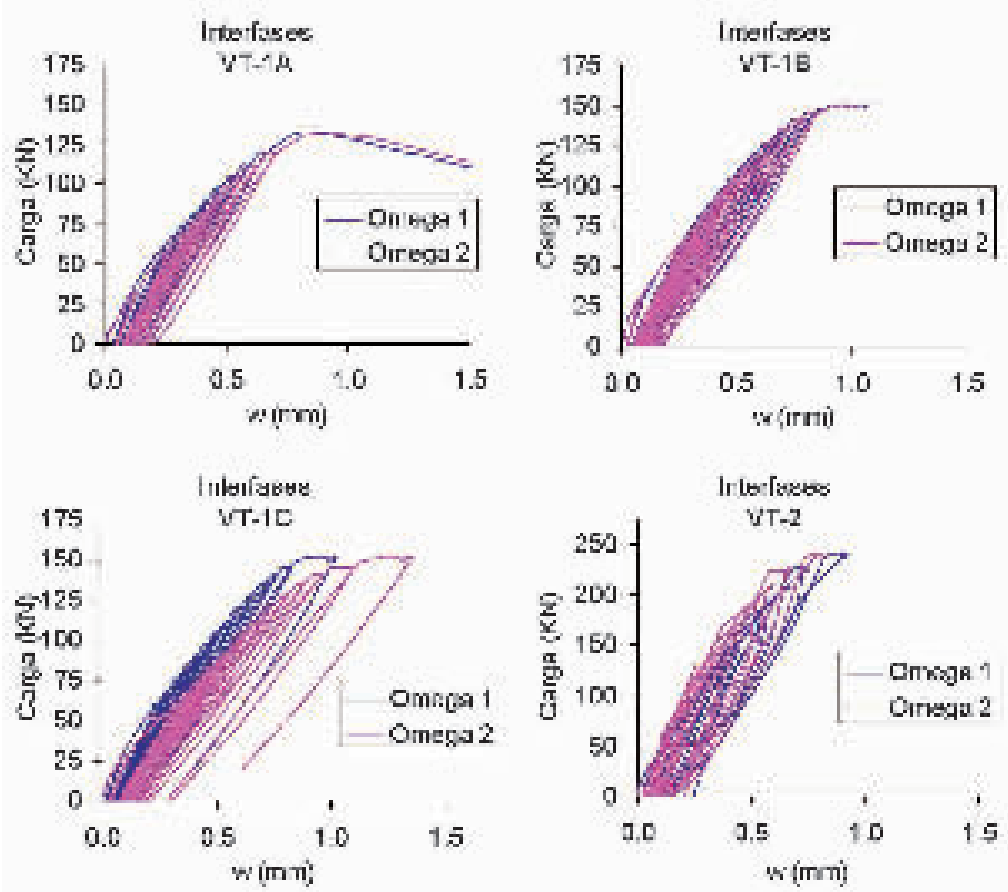

21

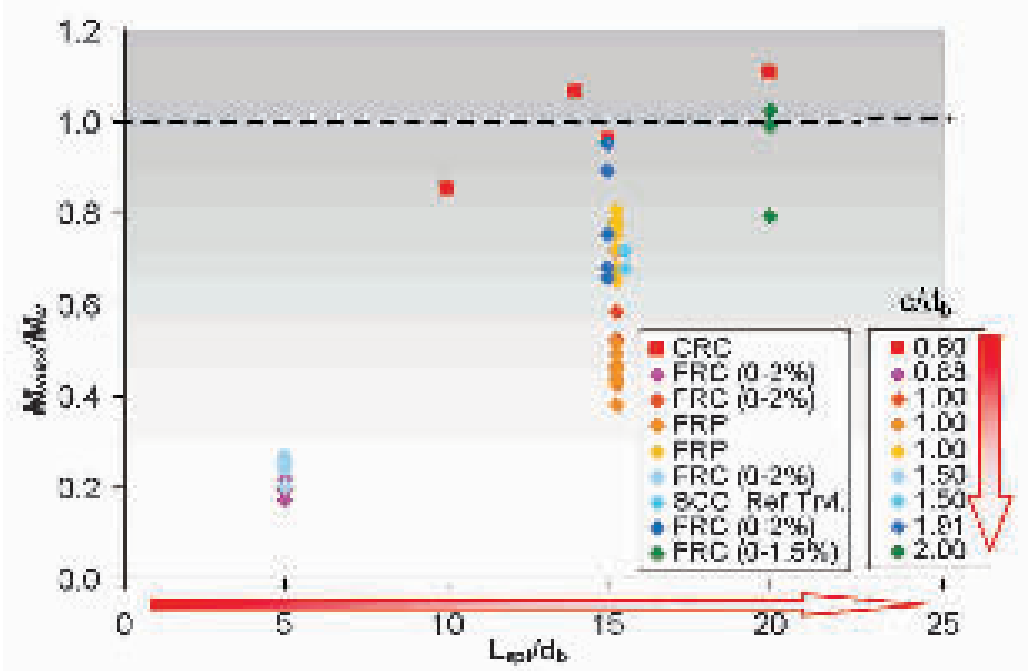

22

Figura 2, donde el uso de placas metálicas soldadas presentan las dificultades inherentes al proceso de instalación y ejecución propias de las actividades de soldadura. Igualmente los resultados obtenidos en el desarrollo de este estudio son satisfactorios en comparación con los resultados de las investigaciones adelantadas por Hamad et al. (9), Tabla 4, sobre el uso de FRP como medio confinante en zonas de adherencia crítica. El elemento VT-2 desarrolló su capacidad ultima con una longitud de solape de tan sólo $14 \mathrm{db}$ y sin el uso de cercos de refuerzo trasversal en la zona de solape, presentando una considerable fisuración en el hormigón convencional y evidente deflexión antes de la rotura.
21. Amplitud de la fisura en la interfase hormigón convencionalUHPFRC. Elementos VT-1A, VT-1B, VT-1C y VT-2.

22. $M_{\text {máx }} / M_{u}-L_{s l p} / d_{b}$ solapes en ele-

Tabla 5

Longitudes de solape mínimas requeridas según diferentes instrucciones

\begin{tabular}{|l|c|}
\hline & $\mathbf{L}_{\text {solape }} / \mathbf{d}_{\mathbf{b}}$ \\
\hline EHE-08 & 40 \\
\hline CM-90 & 38 \\
\hline
\end{tabular}
mentos a flexión. 


\section{CONCLUSIONES Y RECOMENDACIONES}

Basado en el estudio y comparación de los modos de fallo, el comportamiento cargaflecha, las cargas últimas, las deformaciones en la cuerda inferior y las tensiones de adherencia de los elementos con solape de armadura embebidos en el UHPFRC utilizado en este estudio, pueden mencionarse las siguientes conclusiones:

1. Solamente el elemento VT-1A presentó un fallo típico de adherencia debido al splitting lateral, siendo la plastificación del acero y el aumento de la flecha los condicionantes de la rotura en los restantes elementos; en un modo combinado flexión-splitting para el elemento VT-1B y mediante el desprendimiento derivado de la curvatura para los elementos VT-1C y VT-2.

2. El comportamiento de la región central conformada con UHPFRC, sometida a momento constante, presentó una deformación bastante uniforme en comparación con el comportamiento de las zonas de hormigón convencional, donde se presentaba la iniciación y propagación de fisuras localizadas. La fisuración en la región de UHPFRC se hizo evidente para niveles de carga cercanos al 50\% de la carga última, con fisuras de poca amplitud y profundidad en virtud del comportamiento post-fisuración de los UHPFRC.

3. La capacidad de adherencia en elementos con recubrimientos o separación transversal entre solapes limitada, c/db menor de 0,7 , se mantuvo en niveles satisfactorios en comparación con resultados experimentales de elementos con recubrimientos normales, verificando la efectividad del confinamiento derivado de la acción del material con fibras.

4. El uso de UHPFRC en la zona de solape de armaduras permite el desarrollo de un mayor porcentaje de la capacidad a flexión de los elementos en relación al uso de hormigones y FRC convencionales, incluso con el uso de recubrimientos escasos. Para el caso particular del UHPFRC utilizado en este estudio, en ausencia de estribos cerrados o tirantes verticales en la región del solape y con recubrimientos y separación entre solapes escasas, los elementos con longitud de solape entre $14 \mathrm{db}$ y $20 \mathrm{db}$ presentaron la plastificación de la armadura traccionada y desarrollaron entre un $96 \%$ y $111 \%$ de la capacidad última a flexión de la sección. Considerando el uso de recubrimientos y separaciones trasversales entre solapes cercanos habitualmente en la práctica el uso de longitudes de solape cercanas a los 10 db podría ser incluso viable, requiriéndose sin embargo la comprobación experimental.

5. El procedimiento de carga, con ciclos de carga y descarga completa, resulta bastante exigente para los mecanismos de adherencia, siendo no obstante el comportamiento de los solapes embebidos en UHPFRC ensayados satisfactorio en relación al comportamiento de solapes en hormigón convencional y bajo carga monótona. Trabajos posteriores bajo condiciones que respondan a ciclos de carga que reproduzcan las condiciones de servicio deben realizarse para evaluar las fisuras remanentes y los medios adecuados para el control de la apertura de la fisura en la interfase.

6. Un incremento en la tensión de adherencia al momento del fallo por splitting $u_{s p}$ se evidencia, incluso bajo las exigentes condiciones de recubrimiento y separación de solape utilizadas. Un estudio más detallado de $u_{s p}$ mediante el uso de ensayos a flexión en vigas requiere el uso de elementos con longitudes de solape inferiores a $14 \mathrm{db}$ para obtener modos de fallo por splitting sin la plastificación previa del acero de refuerzo.

7. El uso de UHPFRC constituye una alternativa viable, de simple construcción y fiable para mejorar el comportamiento de conexiones donde no se disponga del espacio suficiente para el solape de la armadura debido a limitaciones en las dimensiones de los elementos y a la congestión en el armado.

8. El uso de CRC y otros UHPFRC y su efectividad para mejorar el comportamiento en zonas de adherencia crítica invitan a la reevaluación y desarrollo de configuraciones innovadoras para la conexión de elementos prefabricados, planteamiento seguido en la investigación actualmente en curso sobre el desarrollo de una tipología de conexión viga-pilar para elementos prefabricados.

\section{AGRADECIMIENTOS}

La colaboración y soporte por parte del IETCC-CSIC, ACHE, Grupo PRAINSA, CRC Technology y ANDECE han sido valiosos para el desarrollo de este trabajo y son sinceramente agradecidos por los autores. 


\section{BIBLIOGRAFÍA}

(1) CEB-FIB: "Código modelo CEB-FIP 1990 para hormigón estructural". Ed., Colegio de Ingeniero de Caminos, Canales y Puertos; GEHO-CEB; Grupo español del Hormigón; Asociación Técnica Española del Pretensado. Madrid, España. 1996.

(2) FIB. Bulletin 10: "Bond of reinforcement in concrete. State of the art". Ed. International federation for structural concrete (fib). Laussane (Switzerland) 427 pp. 2000.

(3) Harajli, M. H.; Mabsout, M. E.: "Evaluation of bond strength of steel reinforcing bars in plain and fiber-reinforced concrete". ACl Structural Journal, Vol. 99, № 4, 2002.

(4) Harajli, M. H.; Salloukh, K. A.: "Effect of fibers on development/splice strength of Reinforcing bars in tension". ACl Materials Journal, Vol. 94, N 4, 1997.

(5) Parra-Montesinos, G. J.; Peterfreund, S. W.; Chao, S.: "Highly damage-tolerant beam-column joints through use of high-performance fiber-reinforced cement composites". ACI Structural Journal, Vol. 102, No 3, 2005.

(6) Fantilli, A. P.; Mihashi, H.; Vallini, P.: "Strain compatibility between HPFRCC and steel reinforcement". Materials and Structures, Vol. 38, No 278, 2005.

(7) Klotz, S.; Holschemacher, K.; Weibe, D.: "Bond of reinforcement in Ultra High Strength Concrete. Ultra High Performance Concrete (UHPC)". Proceedings of the International Symposium on Ultra High Performance Concrete. Kassel, 2004.

(8) Hua, L.: "Improving the performance of precast concrete beam,-to-beam connection with inadequate lap length". Monografia Master, 2007.

(9) Hamad, B.; Rteil, A.; Salwan, B.; Soudki, K.: "Behaviour of bond-critical regions wrapped with fiber-reinforced polymer sheets in normal and high-strength concrete". Journal of Composites for Construction, Vol. 8, 2004.

(10) Aarup, B.; Nielsen, C. V.; Olensen, J. F.: "Effect of fibre on the bond strength of high-strength concrete". Proceedings of $4^{\text {th }}$ International Symposium on Utilization of High-Strength/High-Performance Concrete, París 1996.

(11) Harryson, P.: "High performance joints for concrete bridge applications". Structural Engineering International, Vol. 13, No 1, 2003.

(12) CRC Technology Aps. www.crc-tech.com.

(13) Maya, L. F.: "Estudio para el uso de High Performance Fibre Reinforced Cement Composites (HPFRCC) en conexiones de estructuras prefabricadas". Monografía Diploma de Estudios Avanzados, Universidad Politécnica de Madrid. Madrid, 2007.

(14) AENOR. UNE 36035-00: "Barras corrugadas de acero soldable con características especiales de ductilidad para armaduras de hormigón armado". 2000. (238.)

(15) Zanuy, C.: "Análisis seccional de elementos de hormigón armado sometidos a fatiga, incluyendo secciones entre fisuras". Tesis Doctoral, 2008.

(16) AENOR. UNE 13230. "Aplicaciones ferroviarias. Vía. Traviesas de hormigón para plena vía y aparatos". 2003.

(17) Thompson, M. K.; Ledesma, A. L.; Jirsa, J. E.; Breen, J. E.; Klingner, R. E.: "Anchorage behaviour of headed reinforcement. Center for Transportion Research", University of Austin Texas, Report 18553. Austin, 2003.

(18) Harajli, M. H.; Gharzeddine, O.: "Effect of steel fibers on bond performance of steel bars in NSC and HSC under load reversals". Journal of Materials in Civil Engineering, Vol. 19, № 10, 2007.

(19) Türk, K.; Benli, A.; Calayir, Y.: "Bond strength of tension lap-splices in full scale self-compacting concrete beam". Turkish Journal of Engineering and Environmental Sciences, Vol. 32, № 6, 2008.

(20) Harajli, M. H.; Hamad, B.; Karam, K.: "Bond-slip response of reinforcing bars embedded in plain and fiber Concrete". Journal of Material in Civil Engineering, Vol. 14, 2002.

(21) Hamad, B.; Harajli, M. H.; Jumma, G.: "Effect of fiber reinforcement on bond strength of tension lap splices in high-strength concrete". ACI Structural Journal, Vol. 98, N 5, 2001.

(22) "EHE-Instrucción de hormigón estructural". Ministerio de Fomento, 2008

(23) American Concrete Institute: "ACl-318 Building Code Requirements for Structural Concrete and Commentary". 2008. 